\title{
An Easy-to-Use Machine Learning Model to Predict the Prognosis of Patients With COVID-19: Retrospective Cohort Study
}

Hyung-Jun Kim ${ }^{1 *}$, MD; Deokjae Han ${ }^{1 *}$, MD; Jeong-Han Kim² ${ }^{2}$ MD; Daehyun Kim³ ${ }^{3}$ DDS; Beomman Ha ${ }^{4}, \mathrm{MD}, \mathrm{PhD}$; Woong Seog ${ }^{4}$, MD; Yeon-Kyeng Lee ${ }^{5}$, PhD; Dosang Lim ${ }^{5}$, MSc; Sung Ok Hong ${ }^{5}$, PhD; Mi-Jin Park ${ }^{5}$, MSc; JoonNyung $\mathrm{Heo}^{4}, \mathrm{MD}$

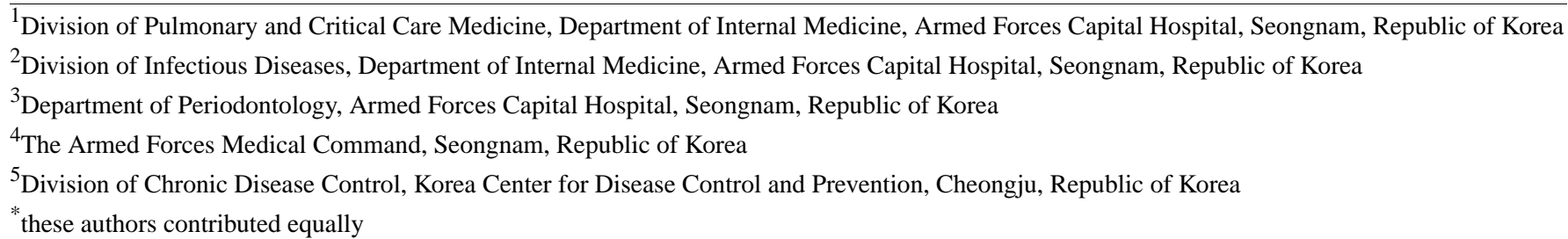

\section{Corresponding Author:}

JoonNyung Heo, MD

The Armed Forces Medical Command

81 Saemaeul-ro 177beon-gil, Bundang-gu

Seongnam,

Republic of Korea

Phone: 82317255490

Email: jnheo@jnheo.com

\begin{abstract}
Background: Prioritizing patients in need of intensive care is necessary to reduce the mortality rate during the COVID-19 pandemic. Although several scoring methods have been introduced, many require laboratory or radiographic findings that are not always easily available.

Objective: The purpose of this study was to develop a machine learning model that predicts the need for intensive care for patients with COVID-19 using easily obtainable characteristics—baseline demographics, comorbidities, and symptoms.

Methods: A retrospective study was performed using a nationwide cohort in South Korea. Patients admitted to 100 hospitals from January 25, 2020, to June 3, 2020, were included. Patient information was collected retrospectively by the attending physicians in each hospital and uploaded to an online case report form. Variables that could be easily provided were extracted. The variables were age, sex, smoking history, body temperature, comorbidities, activities of daily living, and symptoms. The primary outcome was the need for intensive care, defined as admission to the intensive care unit, use of extracorporeal life support, mechanical ventilation, vasopressors, or death within 30 days of hospitalization. Patients admitted until March 20, 2020, were included in the derivation group to develop prediction models using an automated machine learning technique. The models were externally validated in patients admitted after March 21, 2020. The machine learning model with the best discrimination performance was selected and compared against the CURB-65 (confusion, urea, respiratory rate, blood pressure, and 65 years of age or older) score using the area under the receiver operating characteristic curve (AUC).

Results: A total of 4787 patients were included in the analysis, of which 3294 were assigned to the derivation group and 1493 to the validation group. Among the 4787 patients, 460 (9.6\%) patients needed intensive care. Of the 55 machine learning models developed, the XGBoost model revealed the highest discrimination performance. The AUC of the XGBoost model was 0.897 (95\% CI 0.877-0.917) for the derivation group and 0.885 (95\% CI 0.855-0.915) for the validation group. Both the AUCs were superior to those of CURB-65, which were 0.836 (95\% CI 0.825-0.847) and 0.843 (95\% CI 0.829-0.857), respectively.
\end{abstract}

Conclusions: We developed a machine learning model comprising simple patient-provided characteristics, which can efficiently predict the need for intensive care among patients with COVID-19.

(J Med Internet Res 2020;22(11):e24225) doi: 10.2196/24225 


\section{KEYWORDS}

COVID-19; machine learning; prognosis; SARS-CoV-2; severe acute respiratory syndrome coronavirus 2

\section{Introduction}

COVID-19 is an ongoing global pandemic caused by the severe acute respiratory syndrome virus 2 with over 26 million confirmed cases worldwide as of August 31,2020 [1]. The virus is highly transmissible [2] and commonly causes symptoms of fever, cough, fatigue, and myalgia [3]. The mortality rate varies from 0.4 to 304.9 deaths per 1000 COVID-19 cases in the United States according to age group [4], while underlying comorbidities and sex are frequently reported as risk factors for a grave prognosis $[5,6]$.

Other than patient factors, the availability of medical resources is also a major factor for higher risk of death by COVID-19 [7]. The reported case fatality rates are higher in areas with sudden upsurges of COVID-19 compared to other regions, even in the same country. In China, the mortality rates were higher in Hubei Province, in which the outbreak sparked, compared to other provinces [8]. In South Korea, the estimated risk of death was $20.8 \%$ to $25.9 \%$ in Daegu and Gyeongsangbuk-do, which were regions that experienced a sudden COVID-19 outbreak, while other areas had a risk of $1.7 \%$ [9]. Such findings are due to the availability of hospital beds, medical professionals, and other necessary supplies. Therefore, prioritizing patients in need of intensive care is crucial to prevent unnecessary consumption of medical resources by mild or asymptomatic patients.

There have been previous efforts to elucidate the risk factors of grave prognoses among patients with COVID-19 [10-13]. A previous report from China used patient demographics, symptoms, comorbidities, lactate dehydrogenase level, neutrophil-lymphocyte ratio, and radiographic abnormality to predict intensive care unit (ICU) admission, invasive ventilation, or death [10]. Another study from Italy concluded that the proportion of well-aerated lungs was associated with ICU admission or death [13]. Other studies from China also emphasized the use of laboratory findings to predict severe types of COVID-19 [11,12]. Although the performance of these models was excellent, they included laboratory or radiographic findings that may not be quickly available in underdeveloped areas. In addition, rapid adjustment of the scoring systems is not feasible when additional data are collected.

In this study, we aimed to develop a prediction model with information that can easily be provided by patients, limited to baseline demographics, comorbidities, and subjective symptoms. The model aimed to predict the need for intensive care among patients with COVID-19 using an automated machine learning (AutoML) technique [14], which can easily adjust the relative importance of different features as further data become available.

\section{Methods}

\section{Data Source and Study Population}

This was a retrospective study using a nationwide cohort that included all hospitalized patients with COVID-19 in South Korea, developed and managed by the Korean Centers for
Disease Control and Prevention. Patients with laboratory-confirmed COVID-19 were either admitted to a hospital or a community treatment center. The Korean Centers for Disease Control and Prevention requested that all hospitals with patients with COVID-19 register and record their patients' data to the cohort. Data were collected retrospectively through medical chart review by the attending physicians in each center, and were uploaded to an online case report form [15].

Among the patients with COVID-19 hospitalized from January 25,2020 , those who died or were released from quarantine as of June 3, 2020, were included in this study. Patients who were admitted until March 20 were assigned to the derivation group, and those hospitalized after March 21 were assigned to the temporal external validation group. The cut-off point of March 20 was arbitrary. However, two major changes occurred in clinical practice during the study period. First, as testing capacity increased during the pandemic, testing criteria were broadened after February 20. Second, services of community treatment centers commenced on March 2, which we used to quarantine patients with mild symptoms. We excluded patients aged $<18$ years and those with missing data. This study was approved by the Institutional Review Board of the Armed Forces Medical Command (approval number: AFMC-20053-IRB-20-053) with a waiver of informed consent due to the retrospective nature of the study.

\section{Variable Selection}

Variables used for developing the machine learning model included information that could easily be provided by patients without the need for laboratory or radiographic evaluation. The variables were age, sex, smoking history, body temperature, underlying comorbidities, activities of daily living (ADL), and symptoms reported by the patients. Comorbidities included diabetes, heart failure, hypertension, asthma, chronic obstructive pulmonary disease, chronic kidney disease, cancer, chronic liver disease, chronic neurological disorders, chronic hematologic disorders, HIV infection, autoimmune diseases, dementia, and pregnancy. The ADL scale was divided into three categories: independent, partially dependent, and totally dependent. Symptoms considered in the cohort were mental status, cough, sputum, hemoptysis, sore throat, rhinorrhea, chest discomfort, myalgia, arthralgia, fatigue, dyspnea, anosmia, headache, vomiting, and diarrhea.

The CURB-65 score, which stands for confusion, urea, respiratory rate, blood pressure, and 65 years of age or older, was chosen as a comparison against the machine learning model [16]. The score consists of mentality, blood urea nitrogen level, respiratory rate, blood pressure, and age [16]. These data were also extracted from the cohort. Levels of blood urea nitrogen were extracted only to calculate the CURB-65 score and were not included in the machine learning model.

\section{Outcome for the Prediction Models}

The primary outcome was predicting need for intensive care, which we defined as admission to the ICU, use of extracorporeal 
life support, mechanical ventilation, vasopressors, or death during the first 30 days of admission. Information on the use of extracorporeal life support, mechanical ventilation, or vasopressors was included to account for patients who could not be admitted to the ICU due to limited availability.

\section{Machine Learning Analysis}

Complete case analysis was performed, and continuous variables were inspected for input errors. AutoML was used to automate the process of constructing pipelines for the development of the machine learning models, such as hyperparameter optimization and model training. H2O.ai was used to develop these AutoML models [14,17].

The algorithms used during the development of the prediction models using AutoML can be classified into three categories: linear, decision tree based, and neural network based. Linear algorithms are essentially multidimensional linear mathematical formulas. They are intuitive and easy to interpret, and problems that can be described in a linear manner would be best solved by these algorithms. Decision tree-based algorithms consist of a multitude of decision trees comprising multiple true or false conditions for input variables. We used the sum of the decisions made by the decision trees for final classification. These models are better for processing categorical variables with multiple levels, and they can account for interactions between variables. A neural network comprises layers of interconnected artificial neurons that are designed based on a biological neuron. These artificial neurons receive multiple inputs that are multiplied by weights, and they output the sum of these inputs. Neural network models are difficult to interpret, but they can successfully represent complicated interactions between inputs. However, these models are not ideal for representing categorical inputs with multiple levels. Since it is unclear which algorithm can best explain the current problem, all these algorithms were used to develop predictive models, which were then compared based on their discriminative power.

The following models were trained in the AutoML process: 3 prespecified XGBoost gradient boosting machine models, a fixed grid of generalized linear models, a default random forest, 5 prespecified $\mathrm{H} 2 \mathrm{O}$ gradient boosting machines, a near-default deep neural network, an extremely randomized forest, a random grid of XGBoost gradient boosting machines, a random grid of
$\mathrm{H} 2 \mathrm{O}$ gradient boosting machines, and a random grid of deep neural network models. Two stacked ensemble models were developed using the aforementioned developed models [18].

\section{Other Statistical Considerations}

Descriptive statistics were performed for all variables in both derivation and validation groups. Patient characteristics were summarized as counts with proportions for categorical variables and median with interquartile range for continuous variables. Results of the calculated probability based on the machine learning model have been presented in numbers ranging from 0 to 100 , with 0 being the lowest probability of requiring intensive care, and 100 being the highest. The numbers were used to calculate area under the receiver operating characteristic curve (AUC) in the derivation and validation groups. For the derivation group, the mean value of the AUC for the 5 cross-validation sets of each model was used to compare the performance of the developed models. Receiver operating characteristic (ROC) curves were drawn, and the areas under the curves were calculated to assess the predictive performance of the models. $P$ were calculated between the AUC of the machine learning model and the CURB-65 score. Sensitivity, specificity, positive predictive value (PPV), negative predictive value (NPV), and F-measures were measured for different cut-off values. Confusion matrices were constructed for both derivation and validation groups. All $P$ values were two-sided, and a $P$ value of $<.05$ was considered statistically significant. Statistical analysis was performed using R 4.0.0 (The $\mathrm{R}$ Foundation), with the pROC package to draw the ROC curves [19].

\section{Results}

\section{Patient Characteristics}

A total of 5193 patients with polymerase chain reaction-confirmed COVID-19 from 100 centers were registered with the nationwide cohort during the study period. Patients under 18 years $(n=117,2.2 \%)$ and those with missing data $(n=289,5.6 \%)$ were excluded, leaving 4787 patients for analysis. Among these patients, 3294 were assigned to the derivation group, and the remaining 1493 patients were assigned to the validation group (Figure 1). 
Figure 1. Flowchart of the patient selection process. Patients hospitalized in 100 hospitals in South Korea from January 25, 2020, to June 3, 2020, were included. Patients who were admitted until March 20 were assigned to the derivation group, and those hospitalized after March 21 were assigned to the validation group.

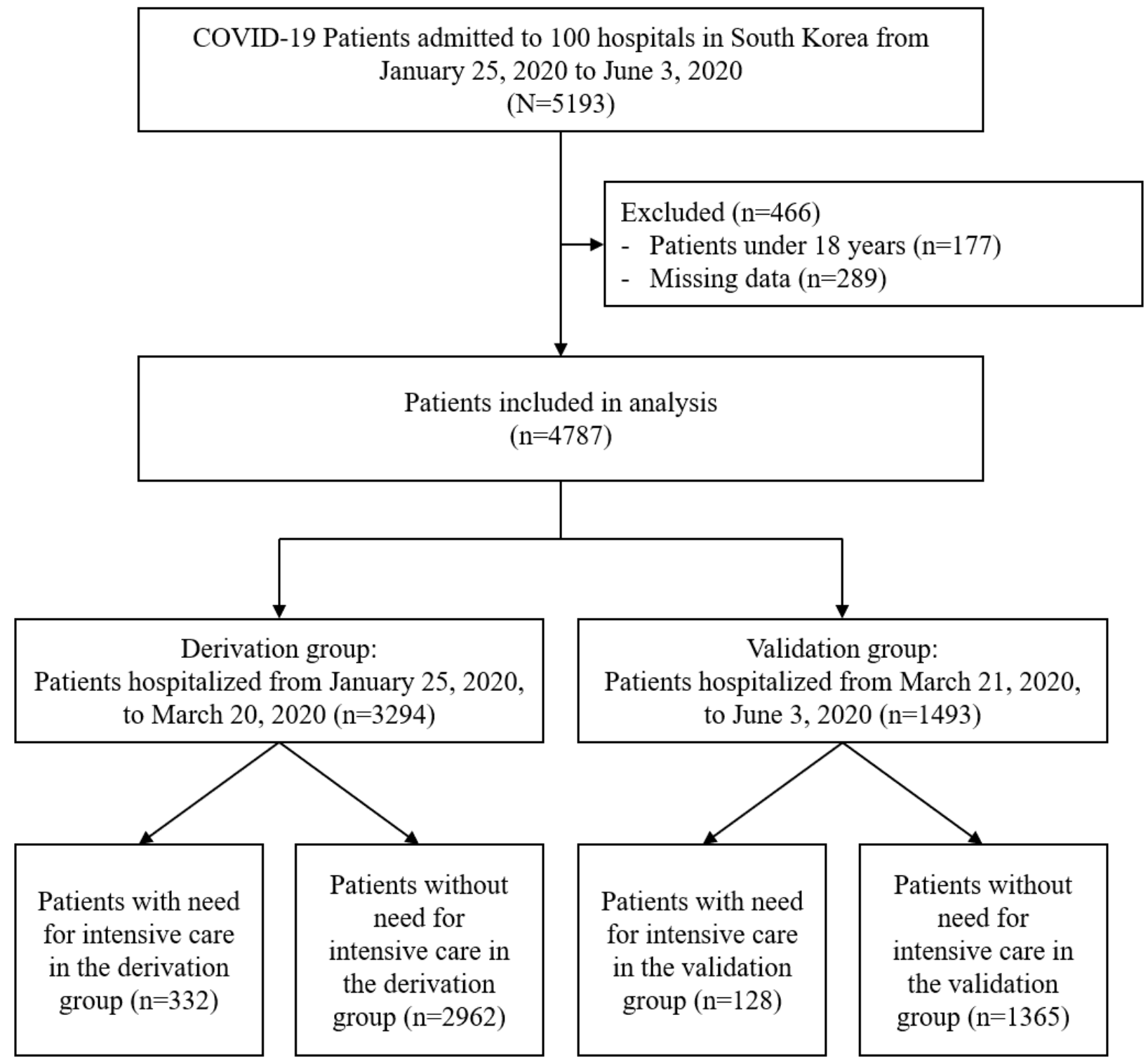

Compared to the patients in the validation group, those in the derivation group were older (median 57.0 years [IQR 42.0-68.0] vs 53.0 years [IQR 30.0-66.0]; $P<.001$ ), with a lower proportion of males $(\mathrm{n}=1227,37.2 \%$ vs $\mathrm{n}=681,45.6 \% ; P<.001)$. Patients in the derivation group experienced more symptoms, including cough $(\mathrm{n}=1537,46.6 \%$ vs $\mathrm{n}=440,29.5 \% ; P<.001)$, sputum $(\mathrm{n}=1054,32.0 \%$ vs $\mathrm{n}=304,20.4 \% ; P<.001)$, headache $(\mathrm{n}=599$, $18.2 \%$ vs $\mathrm{n}=165,11.1 \% ; P<.001)$, and myalgia $(\mathrm{n}=568,17.2 \%$ vs $\mathrm{n}=159,10.7 \% ; P<.001)$, but less anosmia $(\mathrm{n}=40,1.2 \%$ vs $\mathrm{n}=97,6.5 \% ; P<.001)$ compared to the validation group. The presence of underlying comorbidities was largely similar

between the two groups, except for hypertension ( $\mathrm{n}=883,26.8 \%$ vs $\mathrm{n}=351,23.5 \% ; P=.02)$ and diabetes $(\mathrm{n}=537,16.3 \%$ vs $\mathrm{n}=204$, $13.7 \% ; P=.02)$, which were more common in the derivation group, and dementia $(\mathrm{n}=182,5.5 \%$ vs $\mathrm{n}=153,10.3 \% ; P<.001)$, which was more common in the validation group. Patients in the derivation group were more independent in terms of their ADL compared to those in the validation group $(n=2932,89.0 \%$ vs $\mathrm{n}=1188,79.6 \% ; P<.001)$.

A total of 460 patients $(9.6 \%)$ suffered the need for intensive care, of which 221 (4.6\%) patients were admitted to the ICU, and $223(4.7 \%)$ died (Table 1). 
Table 1. Descriptive statistics for the included patients according to derivation and validation groups.

\begin{tabular}{|c|c|c|c|c|}
\hline Variable & Total patients $(\mathrm{N}=4787)$ & Derivation group $(\mathrm{n}=3294)$ & Validation group $(n=1493)$ & $P$ value \\
\hline Age (years), median (IQR) & $55.0(38.0-68.0)$ & $57.0(42.0-68.0)$ & $53.0(30.0-66.0)$ & $<.001$ \\
\hline Sex (male), n (\%) & $1908(39.9)$ & $1227(37.2)$ & $681(45.6)$ & $<.001$ \\
\hline Smoking history, n (\%) & & & & $<.001$ \\
\hline Never smoked & $4388(91.7)$ & 3084 (93.6) & $1304(87.4)$ & N/A ${ }^{a}$ \\
\hline Former smoker & $136(2.8)$ & $97(2.9)$ & $39(2.6)$ & N/A \\
\hline Current smoker & $263(5.5)$ & $114(3.5)$ & $149(10.0)$ & N/A \\
\hline Body temperature $\left({ }^{\circ} \mathrm{C}\right)$, median $(\mathrm{IQR})$ & $36.8(36.5-37.2)$ & $36.9(36.5-37.3)$ & $36.8(36.5-37.2)$ & .002 \\
\hline \multicolumn{5}{|l|}{ Symptoms, $n(\%)$} \\
\hline Cough & $1977(41.3)$ & 1537 (46.6) & $440(29.5)$ & $<.001$ \\
\hline Sputum & $1358(28.4)$ & $1054(32.0)$ & $304(20.4)$ & $<.001$ \\
\hline Headache & $764(16.0)$ & $599(18.2)$ & $165(11.1)$ & $<.001$ \\
\hline Myalgia & $727(15.2)$ & $568(17.2)$ & $159(10.7)$ & $<.001$ \\
\hline Sore throat & $688(14.4)$ & $513(15.6)$ & $175(11.7)$ & .001 \\
\hline Dyspnea & $654(13.7)$ & $543(16.5)$ & $111(7.4)$ & $<.001$ \\
\hline Rhinorrhea & $424(8.9)$ & $318(9.7)$ & $106(7.1)$ & .005 \\
\hline Diarrhea & $399(8.3)$ & $327(9.9)$ & $72(4.8)$ & $<.001$ \\
\hline Chest pain & $369(7.7)$ & $305(9.3)$ & $64(4.3)$ & $<.001$ \\
\hline Nausea/vomiting & $225(4.7)$ & $176(5.3)$ & $49(3.3)$ & .002 \\
\hline Fatigue & $188(3.9)$ & $149(4.5)$ & $39(2.6)$ & .002 \\
\hline Anosmia & $137(2.9)$ & $40(1.2)$ & $97(6.5)$ & $<.001$ \\
\hline Hemoptysis & $26(0.5)$ & $23(0.7)$ & $3(0.2)$ & .051 \\
\hline Altered mentality & $37(0.8)$ & $22(0.7)$ & $15(1.0)$ & .29 \\
\hline Arthralgia & $18(0.4)$ & $16(0.5)$ & $2(0.1)$ & .11 \\
\hline \multicolumn{5}{|l|}{ Comorbidities, n (\%) } \\
\hline Hypertension & $1234(25.8)$ & $883(26.8)$ & $351(23.5)$ & .02 \\
\hline Diabetes & $741(15.5)$ & $537(16.3)$ & $204(13.7)$ & .02 \\
\hline Dementia & $335(7.0)$ & $182(5.5)$ & $153(10.3)$ & $<.001$ \\
\hline Chronic cardiac disease & $195(4.1)$ & $142(4.3)$ & $53(3.6)$ & .25 \\
\hline Cancer & $160(3.3)$ & $113(3.4)$ & $47(3.2)$ & .68 \\
\hline Asthma & $123(2.6)$ & $95(2.9)$ & $28(1.9)$ & .052 \\
\hline Chronic liver disease & $80(1.7)$ & $56(1.7)$ & $24(1.6)$ & .92 \\
\hline Heart failure & $70(1.5)$ & $44(1.3)$ & $26(1.7)$ & .34 \\
\hline Chronic kidney disease & $60(1.3)$ & $48(1.5)$ & $12(0.8)$ & .08 \\
\hline Chronic obstructive pulmonary disease & $42(0.9)$ & $35(1.1)$ & $7(0.5)$ & .06 \\
\hline Chronic neurologic disorder & $42(0.9)$ & $24(0.7)$ & $18(1.2)$ & .14 \\
\hline Chronic hematologic disorder & $35(0.7)$ & $28(0.8)$ & $7(0.5)$ & .21 \\
\hline Autoimmune disease & $34(0.7)$ & $27(0.8)$ & $7(0.5)$ & .25 \\
\hline Pregnancy & $20(0.4)$ & $13(0.4)$ & $7(0.5)$ & .90 \\
\hline HIV infection & $10(0.2)$ & $7(0.2)$ & $3(0.2)$ & $>.99$ \\
\hline Activities of daily living, $n(\%)$ & & & & $<.001$ \\
\hline Independent & $4120(86.1)$ & $2932(89.0)$ & 1188 (79.6) & N/A \\
\hline Partially dependent & $375(7.8)$ & $203(6.2)$ & $172(11.5)$ & N/A \\
\hline
\end{tabular}




\begin{tabular}{|c|c|c|c|c|}
\hline Variable & Total patients $(\mathrm{N}=4787)$ & Derivation group $(\mathrm{n}=3294)$ & Validation group $(\mathrm{n}=1493)$ & $P$ value \\
\hline Totally dependent & $292(6.1)$ & $160(4.9)$ & $132(8.8)$ & N/A \\
\hline Need for intensive care, $n(\%)$ & $460(9.6)$ & $332(10.1)$ & $128(8.6)$ & .12 \\
\hline Death & $223(4.7)$ & $161(4.9)$ & $62(4.2)$ & .30 \\
\hline Admission to $\mathrm{ICU}^{\mathrm{b}}$ & $221(4.6)$ & $169(5.1)$ & $52(3.5)$ & .02 \\
\hline Vasopressor treatment & $119(2.5)$ & $84(2.5)$ & $35(2.3)$ & .75 \\
\hline Mechanical ventilation & $66(1.4)$ & $54(1.6)$ & $12(0.8)$ & .001 \\
\hline Extracorporeal life support & $27(0.6)$ & $21(0.6)$ & $6(0.4)$ & .43 \\
\hline
\end{tabular}

${ }^{\mathrm{a}} \mathrm{N} / \mathrm{A}$ : not applicable.

${ }^{\mathrm{b}} \mathrm{ICU}$ : intensive care unit.

\section{Derivation and Internal Validation of the Machine Learning Model}

With the AutoML, 55 machine learning models were developed to predict the need for intensive care among patients with COVID-19. The XGBoost model, which showed an AUC of 0.897 (95\% CI 0.877-0.917) by cross-validation in the derivation group, was chosen as the best machine learning model (Multimedia Appendix 1). The important features of this model were ADL, age, dyspnea, initial body temperature, sex, and underlying comorbidities. More detailed information on each feature is presented in Multimedia Appendix 2. The developed machine learning model revealed significantly better discrimination performance than the CURB-65 score (AUC 0.836 with $95 \%$ CI $0.825-0.847, P<.001)$ for predicting the need for intensive care among patients with COVID-19. A comparison of the ROC curves for the XGBoost machine learning model and the CURB-65 score is shown in Figure 2A.

Figure 2. Receiver operating characteristic curves for the machine learning model (XGBoost) and the CURB-65 (confusion, urea, respiratory rate, blood pressure, and 65 years of age or older) score for predicting patients requiring intensive care. (A) Comparison in the derivation group, where the area under the receiver operating characteristic (AUC) curves were 0.897 for the gradient boosting machine model, and 0.836 for the CURB-65 score $(P<.001)$. (B) Comparison in the temporal external validation group, where the AUC were 0.885 for the machine learning model, and 0.843 for the CURB-65 score $(P=.01)$.

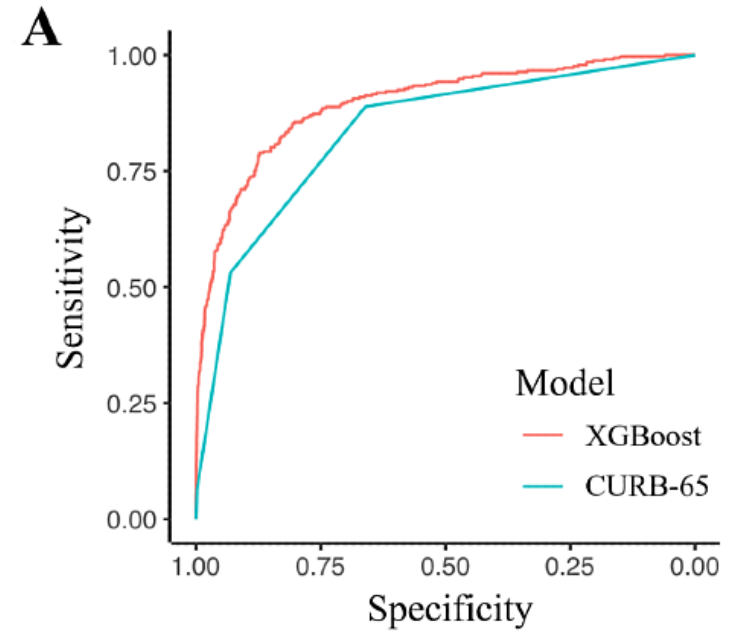

\section{External Validation of the Model}

External validation was performed in the validation group, using the developed XGBoost machine learning model. The discrimination performance of the machine learning model showed an AUC of 0.885 with $95 \%$ CI $0.855-0.915$, which was significantly higher than that of CURB-65 (0.843, 95\% CI 0.829-0.857, $P=.01$ ) (Figure 2B).

\section{Comparison of the Machine Learning Model With the CURB-65 Score With Different Thresholds}

With a cut-off value of 0.5 , the CURB-65 score showed a sensitivity of 0.89 , specificity of 0.66 , PPV of 0.05 , NPV of

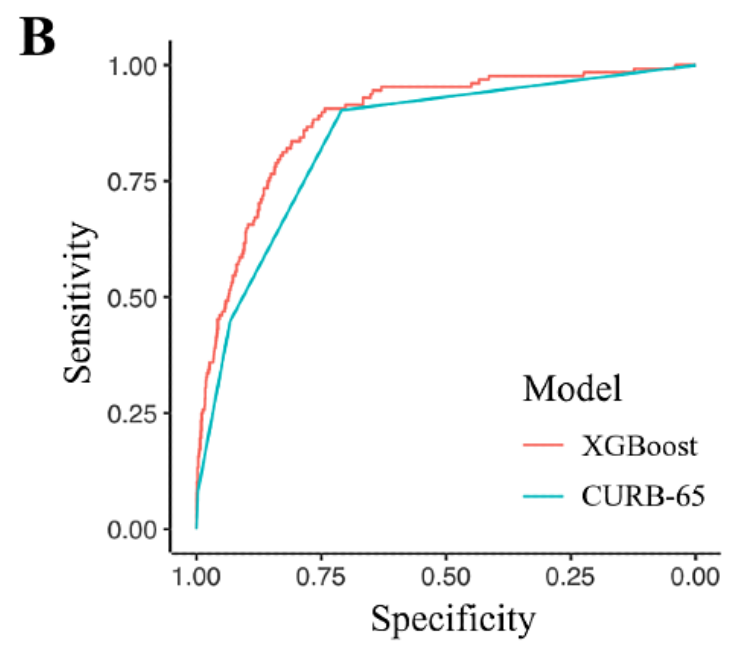

1.00, and F-measure of 0.10. A cut-off value of 0.06 for the XGBoost machine learning model, which shows a similar sensitivity (0.89), revealed a specificity of 0.75 , PPV of 0.36 , NPV of 0.99, and F-measure of 0.43. The XGBoost score also revealed better specificity, PPV, and F-measure compared to CURB-65 when different cut-off thresholds were used (Table 2 ). The confusion matrices of the developed model for the development and validation groups are shown in Multimedia Appendix 3. 
Table 2. Sensitivity, specificity, positive predictive value (PPV), negative predictive value (NPV), and F-measure for the machine learning model and the CURB-65 (confusion, urea, respiratory rate, blood pressure, and 65 years of age or older) score, with different cut-offs.

\begin{tabular}{llllll}
\hline Cut-off & Sensitivity & Specificity & PPV & NPV & F-measure \\
\hline CURB-65 score $>0.5$ & 0.89 & 0.66 & 0.05 & 1.00 & 0.10 \\
XGBoost score $>0.06$ & 0.89 & 0.75 & 0.36 & 0.99 & 0.43 \\
CURB-65 score $>1.5$ & 0.53 & 0.93 & 0.14 & 0.99 & 0.22 \\
XGBoost score $>0.34$ & 0.53 & 0.97 & 0.93 & 0.95 \\
CURB-65 score $>2.5$ & 0.06 & 1.00 & 0.95 & 0.90 & 0.11 \\
XGBoost score $>0.89$ & 0.06 & 1.00 & 0.90 & 0.12 \\
\hline
\end{tabular}

\section{Web Application of Prediction Models}

A web-based application was developed for better accessibility and easy use of the models. The application can be accessed online [20] (Figure 3), and it has been enlisted in the World
Health Organization's Digital Health Atlas [21]. The application calculates the probability of need for intensive care, which is computed according to the derived model. However, it does not store any data yet. It is intended for use by medical practitioners to aid with medical decisions.

Figure 3. Screenshots of the web-based application for easy usage of the developed machine learning model [20]. After input of simple patient-derived information, the probability of the need for intensive care within 30 days is calculated.

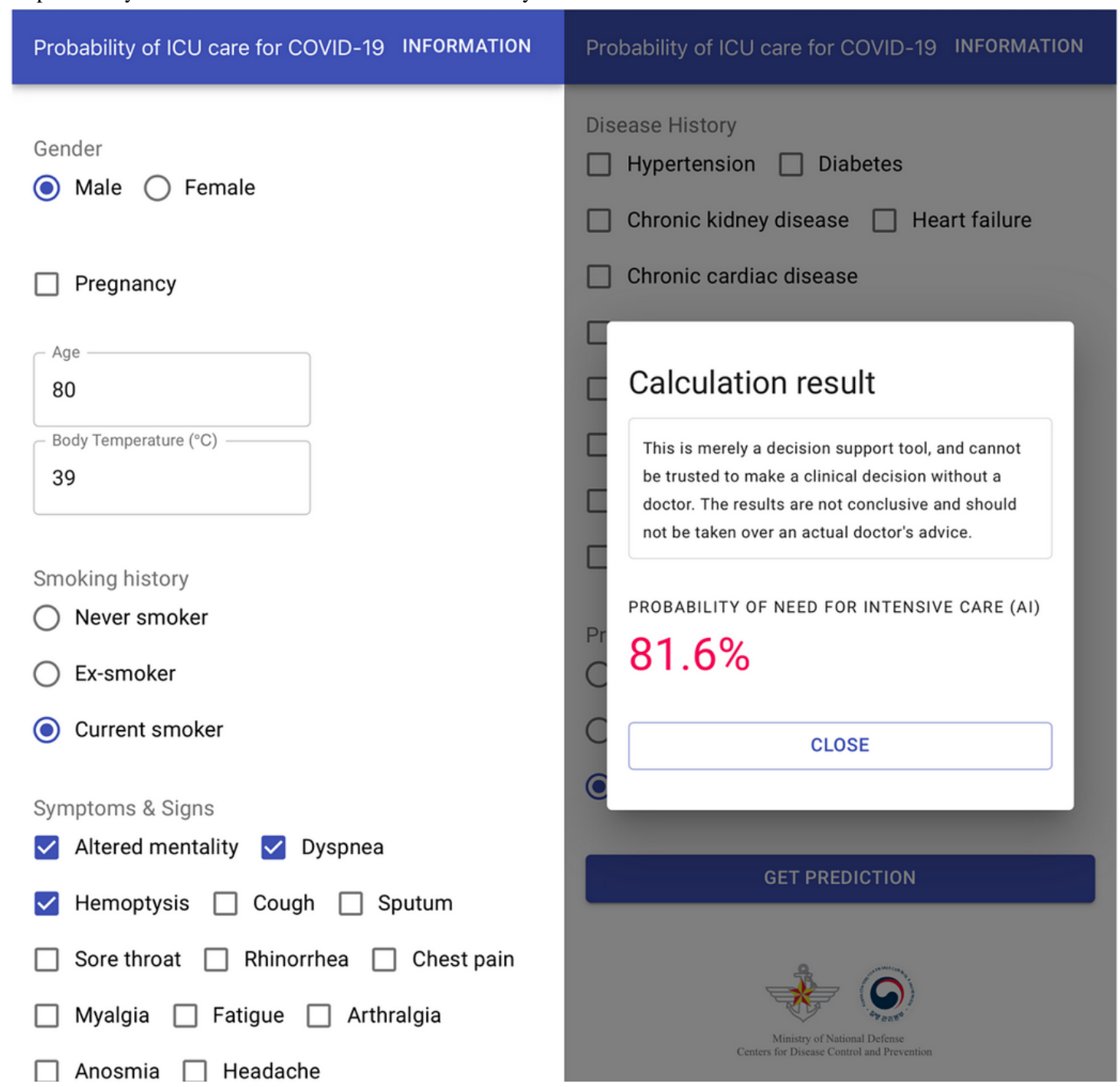

\section{Discussion}

\section{Principal Results}

This study presents a machine learning model that predicts the need for intensive care among patients with COVID-19 from a nationwide cohort in South Korea, including 100 hospitals. The model was derived from the data of patients who were hospitalized from January 25, 2020, to March 20, 2020, and was validated in a separate group of patients hospitalized between March 21, 2020, and June 3, 2020. The AUC of the 
machine learning model was 0.897 (95\% CI 0.877-0.917) for the derivation cohort and 0.885 (95\% CI 0.855-0.915) for the validation cohort, which revealed better discrimination performance than that of CURB-65. Important features included ADL, age, dyspnea, initial body temperature, and sex.

\section{Comparison With Prior Work}

The main features selected in the machine learning model are mostly coherent with previous reports. Older age and male sex have been constantly emphasized as major risk factors for adverse outcomes in patients with COVID-19 [6,22,23]. An early report on 85 fatal cases of COVID-19 in Wuhan [24] revealed that the mean age of patients was 65.8 years, and 62 of the 85 patients (72.9\%) were male [5]. Dyspnea was also a major factor in our study. Incidence of dyspnea is relatively low in COVID-19 as compared to other respiratory symptoms, despite the common pneumonic infiltration on chest radiographs [25]. In a recent systematic review that included 43 studies [3], shortness of breath was observed in $49.2 \%$ in patients with critical illness, while bilateral pneumonia was observed in chest computed tomography (CT) images of $91.0 \%$ of patients with the same disease extent. In an earlier study in China, even in severely ill patients, dyspnea was observed in about $37.6 \%$ of the patients [26]. Therefore, the presentation of dyspnea may imply extensive involvement of the lungs, which leads to grave prognoses [5]. Underlying comorbidities were also repeatedly highlighted as major risk factors for poor prognoses of patients with COVID-19. A pooled analysis of COVID-19 reports emphasized that hypertension is associated with an approximately 2.5 -fold increased risk of higher severity and mortality [27]. Another previous study of 174 patients revealed that patients with diabetes were at higher risk of pneumonia, release of tissue injury-related enzymes, and higher rates of inflammatory responses [28]. Such findings are well summarized in a systematic review that included 3027 patients [5]: male sex (pooled odds ratio [OR] 1.76 with 95\% CI 1.41-2.18), age over 65 years (pooled OR 6.06 with 95\% CI 3.98-9.22), dyspnea (pooled OR 4.16 with 95\% CI 3.13-5.53), presence of cardiovascular disease (pooled OR 5.19 with 95\% CI 3.25-8.29), diabetes (pooled OR 3.68 with 95\% CI 2.68-5.03), and hypertension (pooled OR 2.72 with $95 \%$ CI 1.60-4.64) were all significant factors associated with the progression of COVID-19.

In addition to previous reports, ADL limitation and abnormal body temperature were associated with the need for intensive care among patients with COVID-19 in our study. ADL limitation is known to be an independent risk factor for mortality among elderly patients with pneumonia [29,30]. Because most of the poor outcomes occur in the elderly in COVID-19 $[6,22,23]$, it is probable that ADL limitation leads to the need for intensive care. Abnormal body temperature is also a well-known risk factor for grave prognosis in community-acquired pneumonia patients [31].

\section{Strengths of This Study}

Our machine learning prediction model based on simple patient demographics and subjective symptoms can be useful for the early triage of patients in this pandemic situation. First, it uses information that can be easily provided without advanced equipment, such as age, sex, past medical history, and subjective symptoms. Previous scoring systems [10,11,32-35], including a recently reported deep learning model [36], require laboratory or radiographic findings as the main variables. Although such models can be helpful in fully equipped medical facilities, they initially consume a certain amount of medical resources and time. In areas where laboratory exams or CT exams are limited, our scoring model can be an effective solution for earlier triage. Second, because our model is based on the AutoML technique [20], the relative importance of the features can easily be adjusted with the newly acquired patient data. AutoML techniques have been studied extensively [14] and are expected to be useful for many applications, including in the field of health care [17]. AutoML mainly helps in building machine learning pipelines, which requires expertise in machine learning and is time consuming. It is effective when the time or resources necessary for building a high-functioning model are limited. Considering the rapid adaptability of our model, it can be used effectively with populations with different ethnic or regional backgrounds when further data are collected from the similar populations. It is useful in this pandemic situation, where insufficiency of medical resources has been identified as a critical factor in patient survival [7]. Especially in contexts with less than adequate medical staff, the web-based application is easy to use owing to its intuitive interfaces and clear guides, making it possible for the attending physicians to triage patients without adequate medical knowledge about COVID-19.

CURB-65 was used for comparison with the AutoML model in our study. CURB-65 is a well-known score derived and validated for predicting mortality among patients with community-acquired pneumonia [16], and also shows promising performance in patients with COVID-19 [37]. It is comprised of 6 variables: mental status, levels of blood urea nitrogen, respiratory rate, blood pressure, and age. COVID-19 commonly accompanies pneumonia [4]. In a recent systematic review [38], bilateral $(72.9 \%)$ or unilateral $(25.0 \%)$ involvement of chest X-rays was observed among patients with confirmed COVID-19. A large proportion of the involvement is ground-glass opacities $(68.5 \%)$, which are difficult to recognize from simple chest radiographs. In our study, 2050 of the 4787 patients (42.8\%) underwent chest CT evaluation, and among them, 1535 (74.9\%) were recognized to have pneumonic infiltrations.

\section{Recommendations}

Our model can be used as a decision-support system for medical professionals when active monitoring is not possible due to patient overload caused by the lack of availability of medical staff. However, we cannot recommend a uniform cut-off value for patient transfer to higher-level facilities because this decision depends on the local situation. This decision needs to be made considering the availability of beds in higher-level facilities, the rate of regional increase in the number of patients with COVID-19, and the treatment capability of the facility the patient is currently admitted to. Yet, one solid recommendation that can be made is to prioritize the transfer of patients with a higher probability of need for intensive care when feasible.

\section{Limitations}

Our study had several limitations. First, the sample excluded patients assigned to community treatment centers. However, 
assignment to community treatment centers was mostly conducted for quarantining purposes, not for active treatment. When they required active treatment, such patients were transferred to hospitals and were eventually included in this study. Second, our data set was imbalanced, with $9.6 \%$ of the patients requiring intensive care. Third, our initial model was built based on patients from South Korea. Nevertheless, due to the nature of AutoML, the model can be updated easily when further data become available.

\section{Conclusions}

In conclusion, we derived and validated a machine learning prediction model comprising simple patient-provided characteristics. The model included variables that were largely consistent with previous reports, and it can efficiently anticipate deterioration among patients with COVID-19. The model is easy to use and adjust, requires minimal resources, and can be an effective solution for easy triage in areas with a shortage of medical resources. The model can be used for patient monitoring, and also has a potential as a warning system for self-quarantined patients. However, in the future, randomized trials need to be conducted to examine the direct impact of our model on patient survival.

\section{Acknowledgments}

We acknowledge all the health care workers involved in the diagnosis and treatment of patients with COVID-19 in South Korea. We thank the Central Disease Control Headquarters, National Medical Center, and the health information manager of 100 hospitals for their efforts in collecting medical records. We would like to thank Editage for English language editing.

\section{Authors' Contributions}

HJK, DH, and JH contributed to the conception and design of the study. DH, JHK, DK, BH, WS, YKL, DL, SOH, MJP, and JH were responsible for data collection. HJK, DH, and JH contributed to the analysis and interpretation of data. HJK and JH drafted the paper. DH, JHK, DK, BH, YKL, DL, SOH, MJP, and WS revised the paper for important intellectual content. HJK, DH, JHK, DK, BH, WS, YKL, DL, SOH, MJP, and JH approved the final version of the manuscript to be submitted.

\section{Conflicts of Interest}

None declared.

\section{Multimedia Appendix 1}

AUROC curves of the developed machine learning models. [DOCX File, 19 KB-Multimedia Appendix 1]

\section{Multimedia Appendix 2}

Feature importance of the gradient boosting machine model for prediction of patients requiring intensive care. [DOCX File, 16 KB-Multimedia Appendix 2]

\section{Multimedia Appendix 3}

Confusion matrices for the development and validation groups.

[DOCX File, 16 KB-Multimedia Appendix 3]

\section{References}

1. WHO Coronavirus Disease (COVID-19) Dashboard. World Health Organization. 2020 Aug 31. URL: https://covid19. who.int/ [accessed 2020-09-01]

2. Liu Y, Gayle AA, Wilder-Smith A, Rocklöv J. The reproductive number of COVID-19 is higher compared to SARS coronavirus. J Travel Med 2020 Mar 13;27(2) [FREE Full text] [doi: 10.1093/jtm/taaa021] [Medline: $\underline{32052846}$ ]

3. Fu L, Wang B, Yuan T, Chen X, Ao Y, Fitzpatrick T, et al. Clinical characteristics of coronavirus disease 2019 (COVID-19) in China: A systematic review and meta-analysis. J Infect 2020 Jun;80(6):656-665 [FREE Full text] [doi: 10.1016/j.jinf.2020.03.041] [Medline: 32283155]

4. Wiersinga WJ, Rhodes A, Cheng AC, Peacock SJ, Prescott HC. Pathophysiology, Transmission, Diagnosis, and Treatment of Coronavirus Disease 2019 (COVID-19): A Review. JAMA 2020 Aug 25;324(8):782-793. [doi: 10.1001/jama.2020.12839] [Medline: $\underline{\text { 32648899] }}$

5. Zheng Z, Peng F, Xu B, Zhao J, Liu H, Peng J, et al. J Infect 2020 Aug;81(2):e16-e25 [FREE Full text] [doi: 10.1016/j.jinf.2020.04.021] [Medline: 32335169]

6. Zhou F, Yu T, Du R, Fan G, Liu Y, Liu Z, et al. Clinical course and risk factors for mortality of adult inpatients with COVID-19 in Wuhan, China: a retrospective cohort study. The Lancet 2020 Mar 28;395(10229):1054-1062. [doi: 10.1016/S0140-6736(20)30566-3] [Medline: 32171076] 
7. Ji Y, Ma Z, Peppelenbosch MP, Pan Q. Potential association between COVID-19 mortality and health-care resource availability. The Lancet Global Health 2020 Apr;8(4):e480 [FREE Full text] [doi: 10.1016/S2214-109X(20)30068-1] [Medline: 32109372]

8. Zhang Z, Yao W, Wang Y, Long C, Fu X. Wuhan and Hubei COVID-19 mortality analysis reveals the critical role of timely supply of medical resources. J Infect 2020 Jul;81(1):147-178 [FREE Full text] [doi: 10.1016/j.jinf.2020.03.018] [Medline: 32209384]

9. Shim E, Mizumoto K, Choi W, Chowell G. Estimating the Risk of COVID-19 Death During the Course of the Outbreak in Korea, February-May 2020. J Clin Med 2020 May 29;9(6) [FREE Full text] [doi: 10.3390/jcm9061641] [Medline: 32485871]

10. Liang W, Liang H, Ou L, Chen B, Chen A, Li C, China Medical Treatment Expert Group for COVID-19. Development and Validation of a Clinical Risk Score to Predict the Occurrence of Critical Illness in Hospitalized Patients With COVID-19. JAMA Intern Med 2020 Aug 01;180(8):1081-1089 [FREE Full text] [doi: 10.1001/jamainternmed.2020.2033] [Medline: 32396163]

11. Ji D, Zhang D, Xu J, Chen Z, Yang T, Zhao P, et al. Prediction for Progression Risk in Patients with COVID-19 Pneumonia: the CALL Score. Clin Infect Dis 2020 Apr 09 [FREE Full text] [doi: 10.1093/cid/ciaa414] [Medline: 32271369]

12. Gong J, Ou J, Qiu X, Jie Y, Chen Y, Yuan L, et al. A Tool for Early Prediction of Severe Coronavirus Disease 2019 (COVID-19): A Multicenter Study Using the Risk Nomogram in Wuhan and Guangdong, China. Clin Infect Dis 2020 Jul 28;71(15):833-840 [FREE Full text] [doi: 10.1093/cid/ciaa443] [Medline: 32296824]

13. Colombi D, Bodini FC, Petrini M, Maffi G, Morelli N, Milanese G, et al. Well-aerated Lung on Admitting Chest CT to Predict Adverse Outcome in COVID-19 Pneumonia. Radiology 2020 Aug;296(2):E86-E96 [FREE Full text] [doi: 10.1148/radiol.2020201433] [Medline: 32301647]

14. He X, Zhao K, Chu X. AutoML: A Survey of the State-of-the-Art. arXiv. [FREE Full text] Preprint posted online Aug 2, 2019.

15. iCReaT. National Institute of Health. URL: https://icreat.nih.go.kr/ [accessed 2020-09-04]

16. Lim WS, van der Eerden MM, Laing R, Boersma WG, Karalus N, Town GI, et al. Defining community acquired pneumonia severity on presentation to hospital: an international derivation and validation study. Thorax 2003 May;58(5):377-382 [FREE Full text] [doi: 10.1136/thorax.58.5.377] [Medline: 12728155]

17. Waring J, Lindvall C, Umeton R. Automated machine learning: Review of the state-of-the-art and opportunities for healthcare. Artif Intell Med 2020 Apr;104:101822 [FREE Full text] [doi: 10.1016/j.artmed.2020.101822] [Medline: 32499001]

18. AutoML: Automatic Machine Learning. H2O.ai. 2020. URL: https://docs.h2o.ai/h2o/latest-stable/h2o-docs/automl.html [accessed 2020-09-30]

19. Robin X, Turck N, Hainard A, Tiberti N, Lisacek F, Sanchez J, et al. pROC: an open-source package for R and S+ to analyze and compare ROC curves. BMC Bioinformatics 2011 Mar 17;12:77 [FREE Full text] [doi: 10.1186/1471-2105-12-77] [Medline: 21414208]

20. Probability of ICU care for COVID-19. Ministry of National Defence, Centers for Disease Control and Prevention. URL: http://icu.docl.org [accessed 2020-09-04]

21. DOCL Outcome Prediction Platform (Doctors on the Cloud). Digital Health Atlas. URL: https://digitalatlas.who.int/en/ covid-19/projects/1274/published [accessed 2020-09-01]

22. Bhargava A, Fukushima EA, Levine M, Zhao W, Tanveer F, Szpunar SM, et al. Predictors for Severe COVID-19 Infection. Clin Infect Dis 2020 May 30 [FREE Full text] [doi: 10.1093/cid/ciaa674] [Medline: 32472676]

23. Du R, Liang L, Yang C, Wang W, Cao T, Li M, et al. Predictors of mortality for patients with COVID-19 pneumonia caused by SARS-CoV-2: a prospective cohort study. Eur Respir J 2020 May;55(5) [FREE Full text] [doi: 10.1183/13993003.00524-2020] [Medline: 32269088]

24. Du Y, Tu L, Zhu P, Mu M, Wang R, Yang P, et al. Clinical Features of 85 Fatal Cases of COVID-19 from Wuhan. A Retrospective Observational Study. Am J Respir Crit Care Med 2020 Jun 01;201(11):1372-1379 [FREE Full text] [doi: 10.1164/rccm.202003-05430C] [Medline: 32242738]

25. Bertran Recasens B, Martinez-Llorens JM, Rodriguez-Sevilla JJ, Rubio MA. Lack of dyspnea in patients with Covid-19: another neurological conundrum? Eur J Neurol 2020 Apr 17 [FREE Full text] [doi: 10.1111/ene.14265] [Medline: 32301553]

26. Guan W, Ni Z, Hu Y, Liang W, Ou C, He J, China Medical Treatment Expert Group for Covid-19. Clinical Characteristics of Coronavirus Disease 2019 in China. N Engl J Med 2020 Apr 30;382(18):1708-1720 [FREE Full text] [doi: 10.1056/NEJMoa2002032] [Medline: 32109013]

27. Lippi G, Wong J, Henry BM. Hypertension in patients with coronavirus disease 2019 (COVID-19): a pooled analysis. Pol Arch Intern Med 2020 Apr 30;130(4):304-309 [FRE Full text] [doi: 10.20452/pamw.15272] [Medline: 32231171]

28. Guo W, Li M, Dong Y, Zhou H, Zhang Z, Tian C, et al. Diabetes is a risk factor for the progression and prognosis of COVID-19. Diabetes Metab Res Rev 2020 Mar 31:e3319 [FREE Full text] [doi: 10.1002/dmrr.3319] [Medline: 32233013]

29. Torres OH, Muñoz J, Ruiz D, Ris J, Gich I, Coma E, et al. Outcome predictors of pneumonia in elderly patients: importance of functional assessment. J Am Geriatr Soc 2004 Oct;52(10):1603-1609. [doi: 10.1111/j.1532-5415.2004.52492.x] [Medline: 15450034] 
30. Mody L, Sun R, Bradley SF. Assessment of pneumonia in older adults: effect of functional status. J Am Geriatr Soc 2006 Jul;54(7):1062-1067 [FREE Full text] [doi: 10.1111/j.1532-5415.2006.00797.x] [Medline: 16866676]

31. Fine MJ, Auble TE, Yealy DM, Hanusa BH, Weissfeld LA, Singer DE, et al. A prediction rule to identify low-risk patients with community-acquired pneumonia. N Engl J Med 1997 Jan 23;336(4):243-250. [doi: 10.1056/NEJM199701233360402] [Medline: 8995086]

32. Zou X, Li S, Fang M, Hu M, Bian Y, Ling J, et al. Acute Physiology and Chronic Health Evaluation II Score as a Predictor of Hospital Mortality in Patients of Coronavirus Disease 2019. Crit Care Med 2020 Aug 01;48(8):e657-e665 [FREE Full text] [doi: 10.1097/CCM.0000000000004411] [Medline: 32697506]

33. Mahdjoub E, Mohammad W, Lefevre T, Debray M, Khalil A, Study Group§. Admission chest CT score predicts 5-day outcome in patients with COVID-19. Intensive Care Med 2020 Aug;46(8):1648-1650. [doi: 10.1007/s00134-020-06118-y] [Medline: 32468085]

34. Galloway JB, Norton S, Barker RD, Brookes A, Carey I, Clarke BD, et al. A clinical risk score to identify patients with COVID-19 at high risk of critical care admission or death: An observational cohort study. J Infect 2020 Aug;81(2):282-288 [FREE Full text] [doi: 10.1016/j.jinf.2020.05.064] [Medline: 32479771]

35. Sabri A, Davarpanah AH, Mahdavi A, Abrishami A, Khazaei M, Heydari S, et al. Novel coronavirus disease 2019: predicting prognosis with a computed tomography-based disease severity score and clinical laboratory data. Pol Arch Intern Med 2020 Aug 27;130(7-8):629-634 [FREE Full text] [doi: 10.20452/pamw.15422] [Medline: 32500700$]$

36. Liang W, Yao J, Chen A, Lv Q, Zanin M, Liu J, et al. Early triage of critically ill COVID-19 patients using deep learning. Nat Commun 2020 Jul 15;11(1):3543 [FREE Full text] [doi: 10.1038/s41467-020-17280-8] [Medline: 32669540]

37. Satici C, Demirkol MA, Sargin Altunok E, Gursoy B, Alkan M, Kamat S, et al. Performance of pneumonia severity index and CURB-65 in predicting 30-day mortality in patients with COVID-19. Int J Infect Dis 2020 Sep;98:84-89 [FREE Full text] [doi: 10.1016/j.ijid.2020.06.038] [Medline: 32553714 ]

38. Rodriguez-Morales AJ, Cardona-Ospina JA, Gutiérrez-Ocampo E, Villamizar-Peña R, Holguin-Rivera Y, Escalera-Antezana JP, Latin American Network of Coronavirus Disease 2019-COVID-19 Research (LANCOVID-19). Electronic address: https://www.lancovid.org. Clinical, laboratory and imaging features of COVID-19: A systematic review and meta-analysis. Travel Med Infect Dis 2020 Mar 13:101623 [FRE Full text] [doi: 10.1016/j.tmaid.2020.101623] [Medline: 32179124]

\author{
Abbreviations \\ ADL: activities of daily living \\ AUC: area under the receiver operating characteristic curve \\ AutoML: automated machine learning \\ CT: computed tomography \\ CURB-65: confusion, urea, respiratory rate, blood pressure, and 65 years of age or older \\ ICU: intensive care unit \\ NPV: negative predictive value \\ OR: odds ratio \\ PPV: positive predictive value \\ ROC: receiver operating characteristic
}

\author{
Edited by G Eysenbach; submitted 10.09.20; peer-reviewed by A Alasmari, S Syed, A Azzam, T Scherr; comments to author 27.09.20 \\ revised version received 11.10.20; accepted 25.10.20; published 09.11.20 \\ Please cite as: \\ Kim HJ, Han D, Kim JH, Kim D, Ha B, Seog W, Lee YK, Lim D, Hong SO, Park MJ, Heo J \\ An Easy-to-Use Machine Learning Model to Predict the Prognosis of Patients With COVID-19: Retrospective Cohort Study \\ $J$ Med Internet Res 2020;22(11):e24225 \\ URL: http://www.jmir.org/2020/11/e24225/ \\ doi: $10.2196 / 24225$ \\ PMID: 33108316
}

CHyung-Jun Kim, Deokjae Han, Jeong-Han Kim, Daehyun Kim, Beomman Ha, Woong Seog, Yeon-Kyeng Lee, Dosang Lim, Sung Ok Hong, Mi-Jin Park, JoonNyung Heo. Originally published in the Journal of Medical Internet Research (http://www.jmir.org), 09.11.2020. This is an open-access article distributed under the terms of the Creative Commons Attribution License (https://creativecommons.org/licenses/by/4.0/), which permits unrestricted use, distribution, and reproduction in any medium, provided the original work, first published in the Journal of Medical Internet Research, is properly cited. The complete 
bibliographic information, a link to the original publication on http://www.jmir.org/, as well as this copyright and license information must be included. 